Supporting Information

Semiconductor-Insulator (Nano-)Couples with Tunable Properties

Obtained from Asymmetric Modification of Janus Nanoparticles

\author{
Voichita Mihali* and Andrei Honciuc ${ }^{\dagger *}$ \\ Institute of Chemistry and Biotechnology, Zurich University of Applied Sciences, \\ Einsiedlerstrasse 31, 8820 Waedenswil, Switzerland \\ Current address: University of Basel, Department of Chemistry, Mattentrasse24a, 4058 \\ Basel, Switzerland \\ †"Petru Poni” Institute of Macromolecular Chemistry, Electroactive Polymers and \\ Plasmochemistry Laboratory, Aleea Gr. Ghica Voda 41A, Iasi, 700487, Romania \\ *Corresponding author: e-mail: honciuc.andrei@icmpp.ro
}

\title{
Nanoparticle characterization
}

The NPs and JNPs were characterized with the scanning electron microscopy (SEM) (FEI Quanta FEG 250, operating at 5-30 kV accelerating voltage in the secondary electron (SE) mode, in high vacuum mode $\left(3 \times 10^{-6}-1.8 \times 10^{-5} \mathrm{mbar}\right)$. UV-vis-NIR absorption spectra were measured in solid-state using UV-vis-NIR spectrophotometer (Metrohm AG, NIRS XDS MasterLab Analyzer). FTIR analysis was carried out using Perkin Elmer spectrometer (Spectrum 1000) at room temperature over the frequency range of $4000-500 \mathrm{~cm}^{-1}$. The FTIR spectra are compared in the Figure S6. 

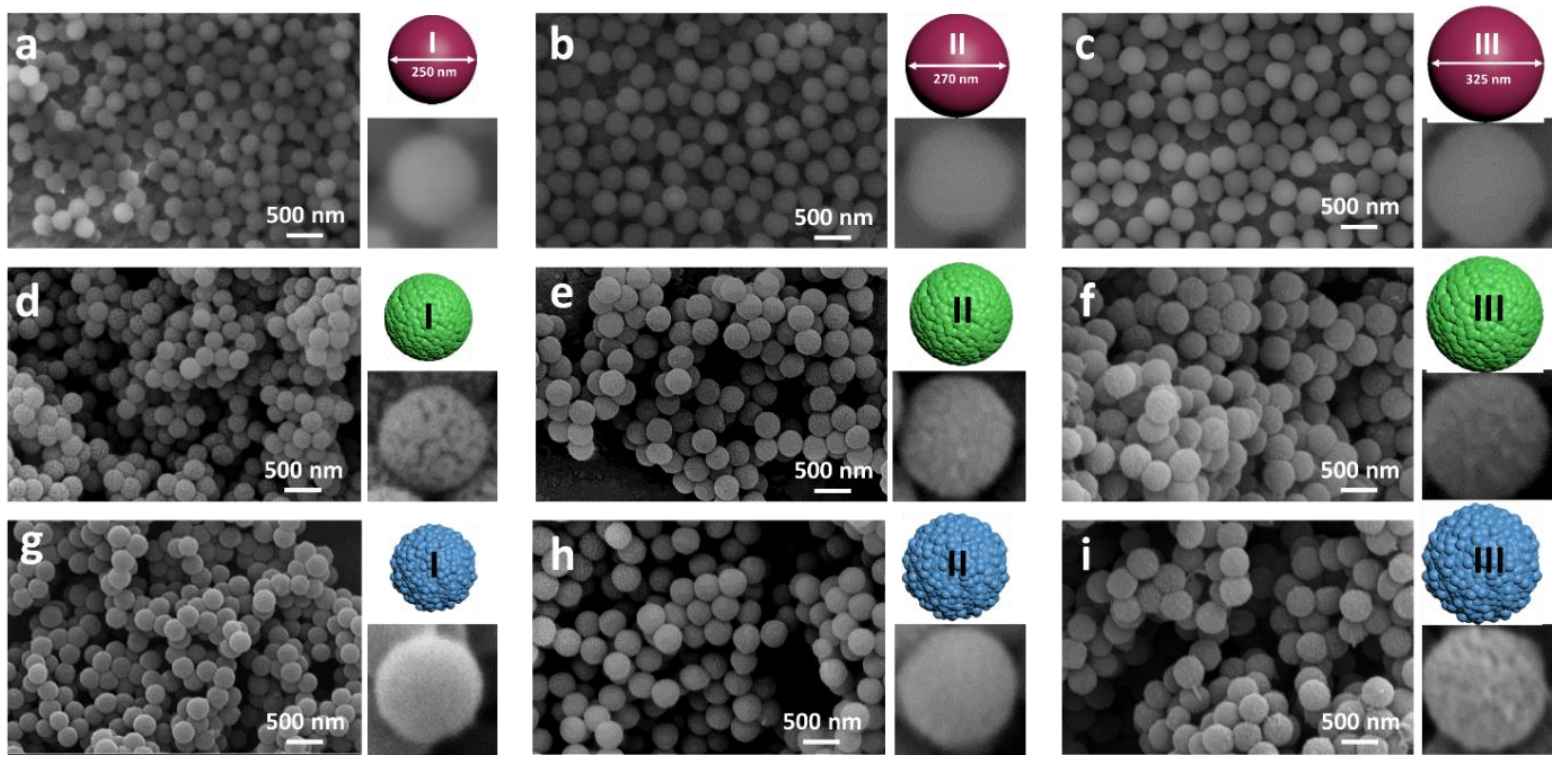

Figure S1. Scanning electron microscopy (SEM) images showing HNPs with different size and the surface modification with PANI and PEDOT/PANI semiconductive polymers: (a) HNPs-I, (b) HNPs-II, (c) HNPs-III, (d) PANI-HNPs-I, (e) PANI-HNPs-II, (f) PANI-HNPs-III, (g) PEDOT/PANI-HNPs-I, (h) PEDOT/PANI-HNPs-II, (i) PEDOT/PANI-HNPs-III.

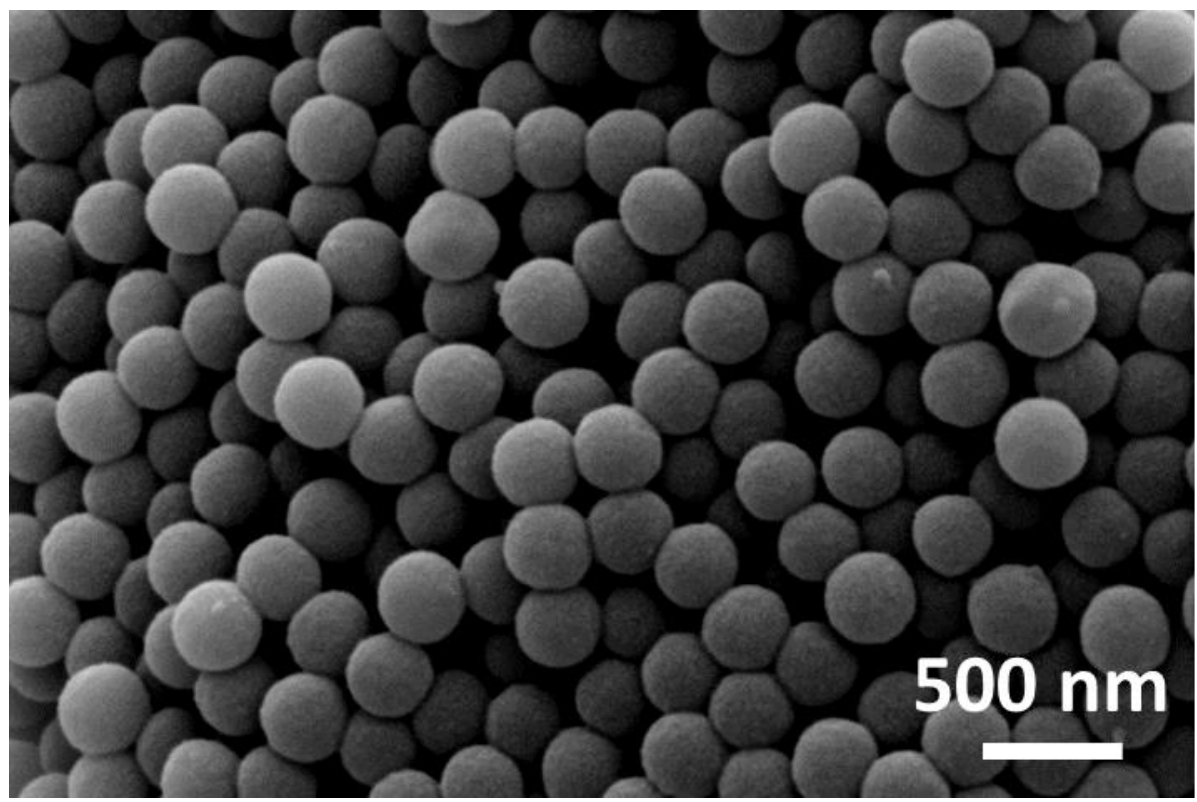

Figure S2. Scanning electron microscopy (SEM) of PEDOT-HNPs-II 

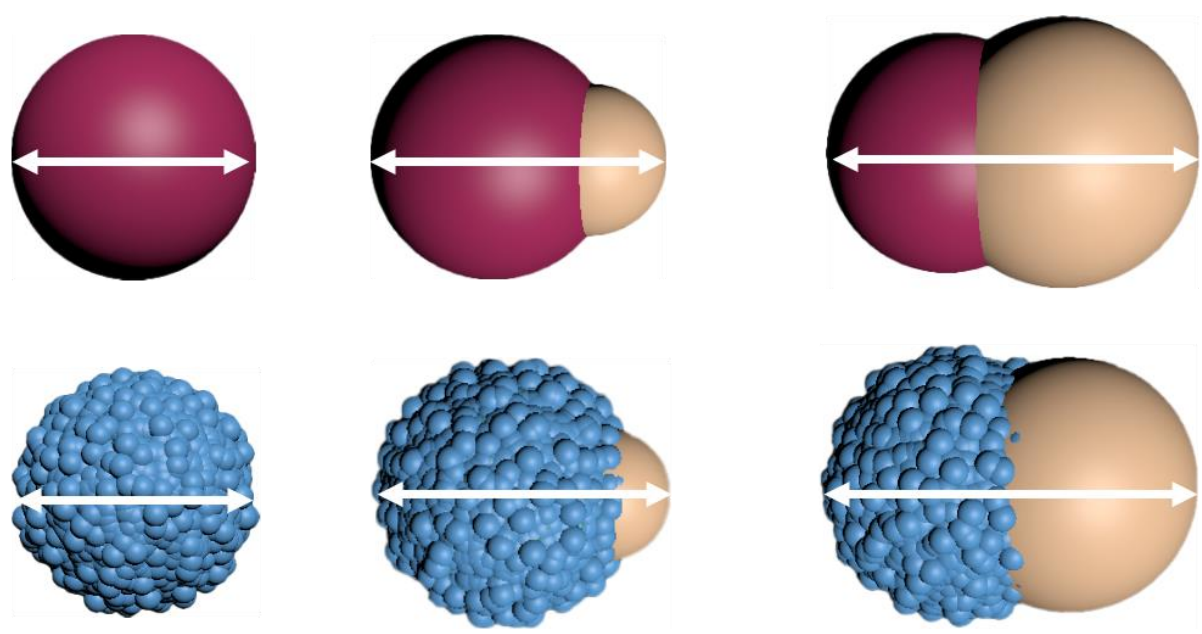

Figure S3. Cartoon depicting the SEM measurement of HNPs and JNPs before and after selective modification with PEDOT/PANI.

\section{Measurement of the electrical conductivity}

Electrical resistance of the NPs was measured using a standard four-point probe technique at room temperature. The particle powder, weighting $75 \mathrm{mg}$, were pressed (10 ton) into pellets (0.04 cm thick, $1.32 \mathrm{~cm}$ diameter) for 4 minutes. Current-voltage (I-V) sweeps of the samples were acquired using a computer controlled Keithley $2182 \mathrm{~A}$ as voltage source and a Fluke 8846A 6.5 digit precision multimeter for the current measurement. The resistance was calculated from the slope of the I-V curves and converted to resistivity $(\Omega \cdot \mathrm{cm})$ and electrical conductivity $(\mathrm{S} / \mathrm{cm})$. The resistivity was calculated from the resistance $\mathrm{R}$ with the formula: $\rho=$ $\frac{\pi \cdot r^{2} \cdot R}{l}$, where the current path length was the same as the thickness of the pellet $(l=0.04 \mathrm{~cm})$ and the cross-sectional area was the area of the pellet where $r$ is the radius of the pellet, $0.65 \mathrm{~cm}$. 


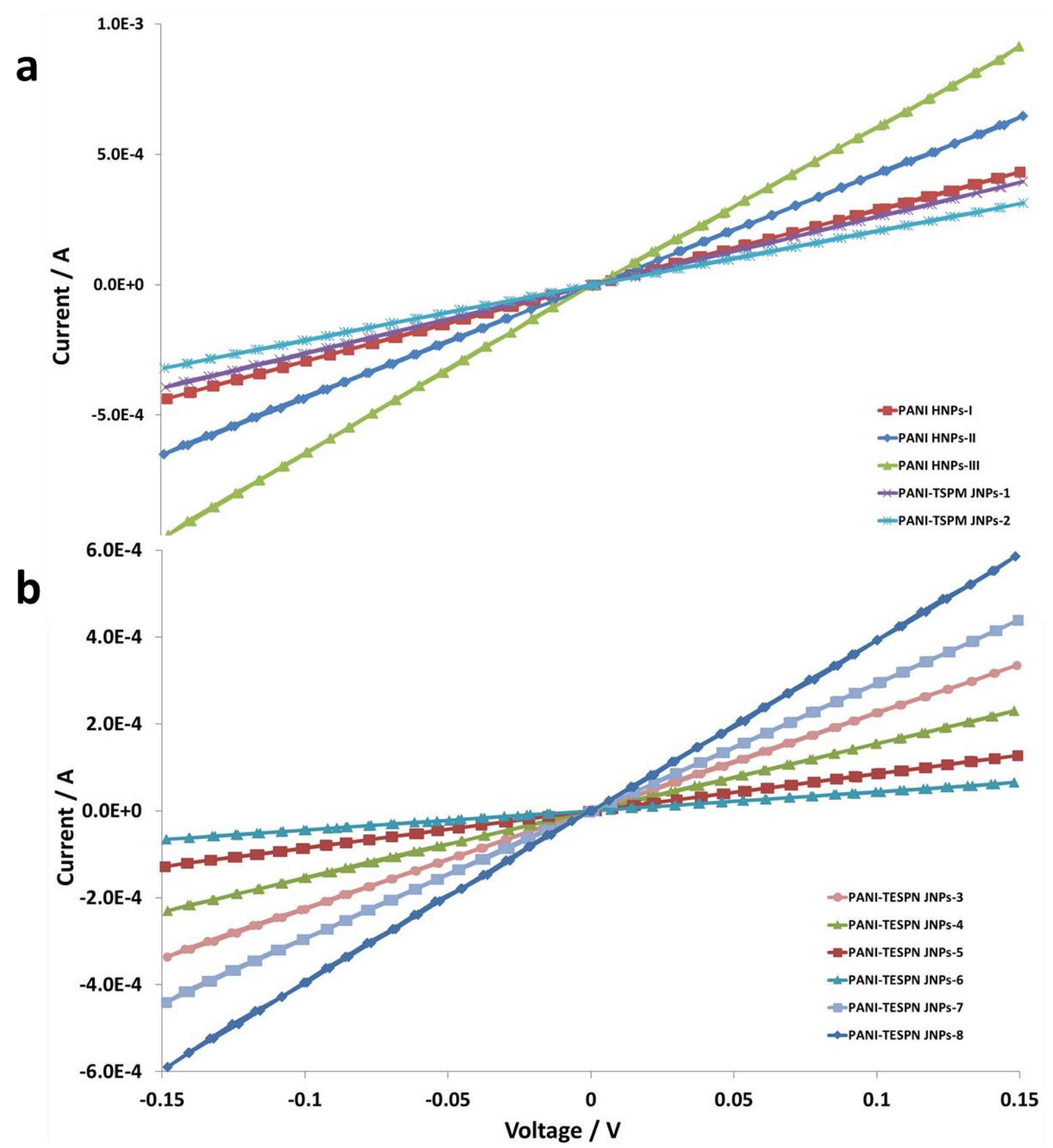

Figure S4. Current- voltage (I-V) sweeps of PANI-HNPs and asymmetrically modified PANI JNPs. The I-V data was acquired on three different samples, the error bars represent the standard deviation, at room temperature on $0.05 \mathrm{~cm}$ thick pellets made in the same conditions for all the samples. 


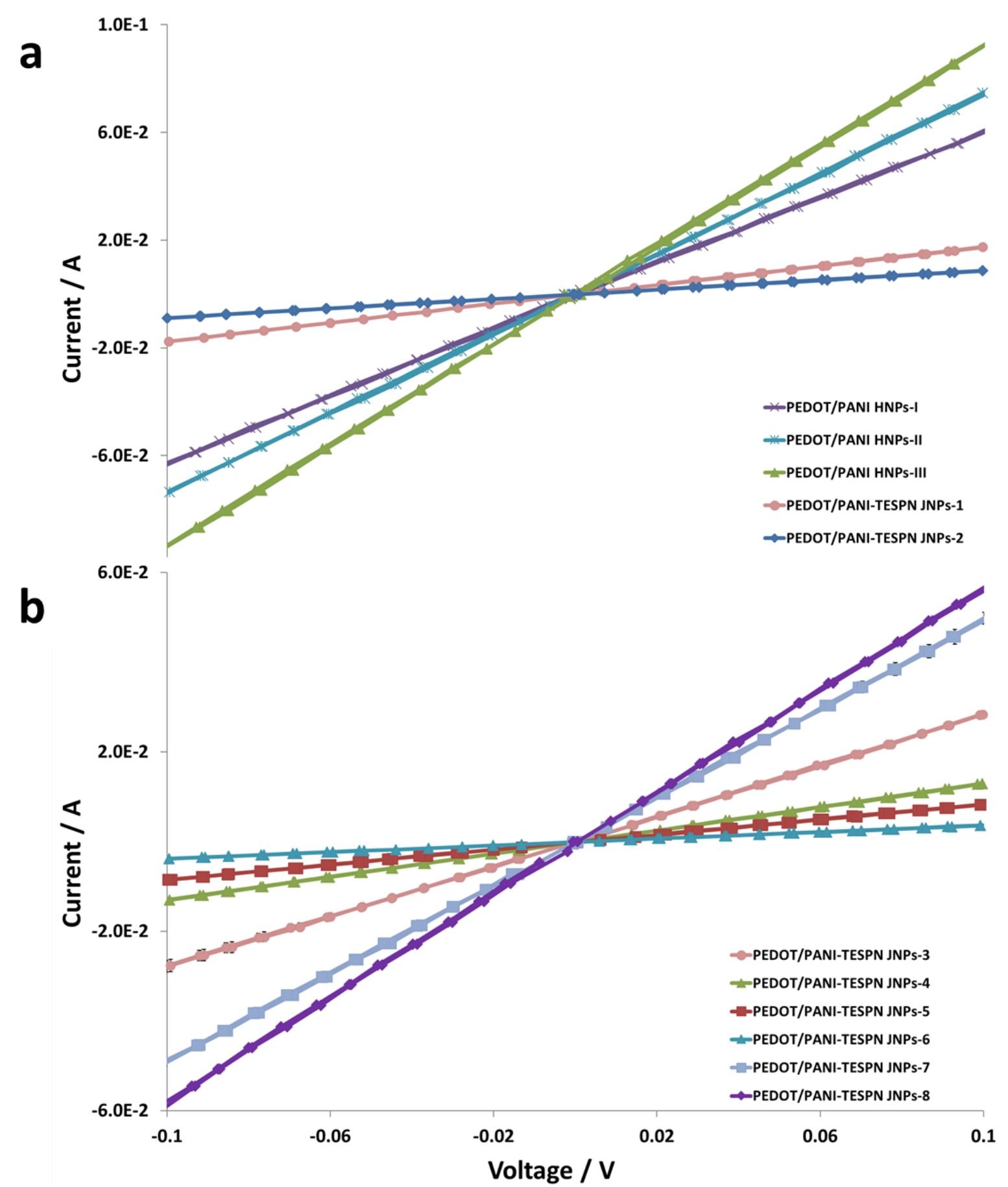

Figure S5. Current- voltage (I-V) sweeps of PEDOT/PANI-HNPs and asymmetrically modified PEDOT/PANI JNPs. The I-V data was acquired on three different samples, the error bars represent the standard deviation, at room temperature on $0.05 \mathrm{~cm}$ thick pellets made in the same conditions for all the samples.

FTIR analysis of the nanoparticles 

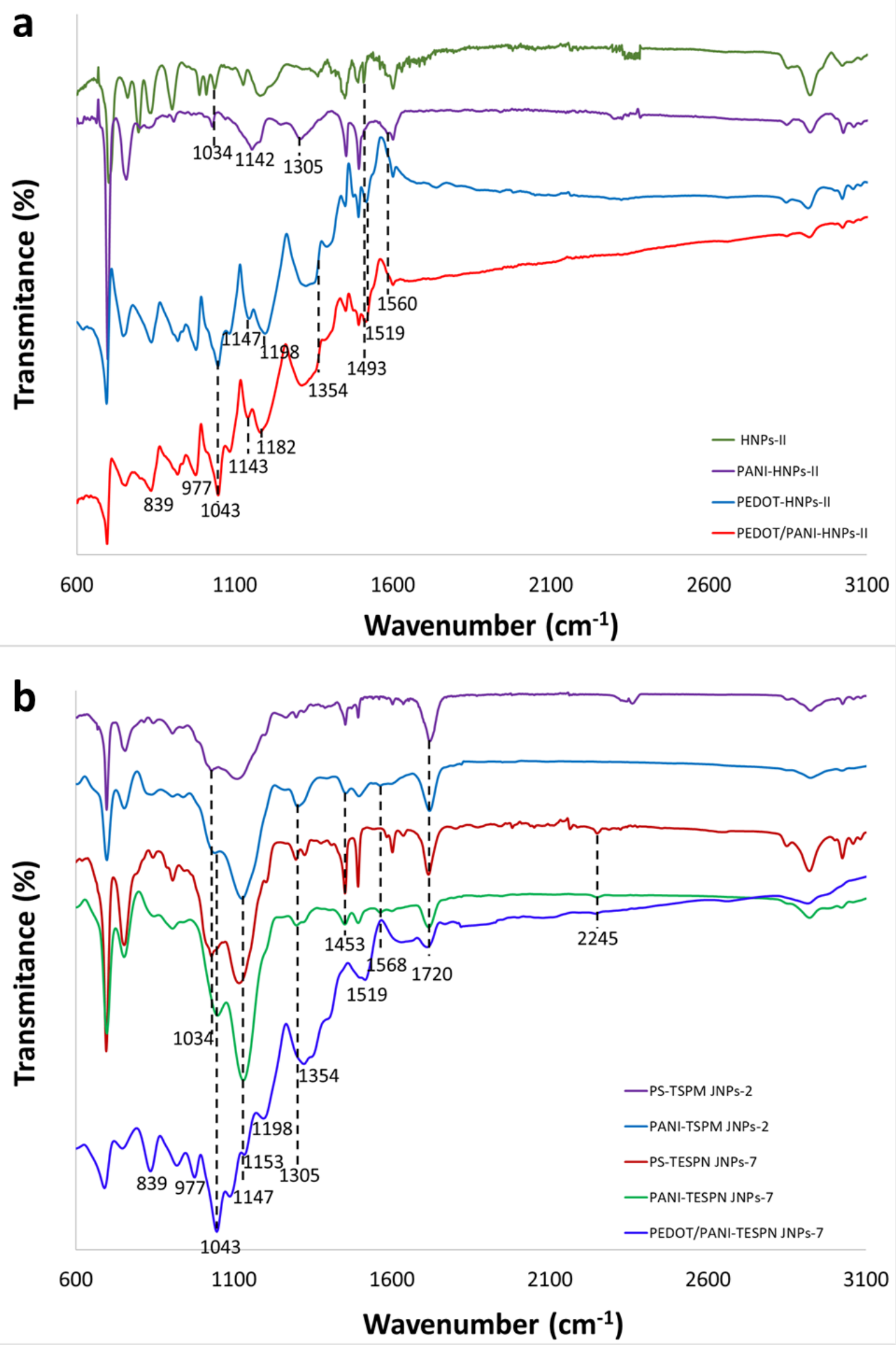

Figure S6. FTIR spectra showing HNPs and JNPs loaded with a PANI layer and PEDOT/PANI bilayer: (a) HNPs, (b) JNPs. 
The FTIR spectra of the unmodified HNPs-II, PANI-HNPs-II and PEDOT/PANIHNPs-II are compared in Figure S6a. For the HNPs-II the peak at $1034 \mathrm{~cm}^{-1}$ can be assigned to the $\mathrm{S}=\mathrm{O}$ stretch in the $-\mathrm{SO}_{3} \mathrm{H}$ group. The presence of the absorption peak at $1142 \mathrm{~cm}^{-1}$ corresponds to the protonated $-\mathrm{NH}^{+}$of PANI.[1] The spectrum of PEDOT-HNPs-II show the typical bands of the PEDOT, such as the peaks at $1354 \mathrm{~cm}^{-1}$ and $1519 \mathrm{~cm}^{-1}$ corresponding to $\mathrm{C}=\mathrm{C}$ and $\mathrm{C}-\mathrm{C}$ stretching of the quinoidal structure of the thiophene ring. The $\mathrm{C}-\mathrm{O}-\mathrm{C}$ bond stretching in the ethylene dioxy group can be observed in the peaks at $1147 \mathrm{~cm}^{-1}$ and $1043 \mathrm{~cm}^{-}$ 1. The peaks observed at 977 and $839 \mathrm{~cm}^{-1}$ it is $\mathrm{C}-\mathrm{S}$ bond stretching in the thiophene ring.[2] The PEDOT/PANI-HNPs-II spectrum strongly resembles that of the PEDOT-HNPs-II suggesting full covering of the thick shell of PEDOT overlayer. Some small differences such as the shift of the ring stretches from 1198 to $1182 \mathrm{~cm}^{-1}$ and from 1147 to $1143 \mathrm{~cm}^{-1}$.

The FTIR spectra of the asymmetrically modified JNPs with PANI layer and PEDOT/PANI semiconducting bilayers are compared in the Figure S6b.

The FTIR spectrum of the unmodified Janus nanoparticles PS-TSPM JNPs-2 and PS-TESPN JNPs-7, exhibit a peak at $1034 \mathrm{~cm}^{-1}$ characteristic to $-\mathrm{SO}_{3}{ }^{-}$group present on the PS lobe and a peak at $1720 \mathrm{~cm}^{-1}$, which corresponds to the $-\mathrm{C}=\mathrm{O}$ vibration in the ester functionality present in the TSPM and the TESPN lobe of the JNPs. The spectrum of PS-TESPN JNPs-7 show the characteristic weak bands at $2245 \mathrm{~cm}^{-1}$ for $\mathrm{CN}$ stretching[3] and this peak is present also in the selectively modified JNPs with PANI and PEDOT/PANI, which confirms the asymmetric functionalization of JNPs.

In the spectra of asymmetrically modified JNPs, the absorption peaks of semiconducting polymer are partly overlapped by the bands of JNPs, but there are still some distinguishing features. The characteristic PANI peaks at 1568 and $1453 \mathrm{~cm}^{-1}$ are attributed to the $\mathrm{C}=\mathrm{C}$ stretching of quinoid and benzenoid rings, respectively.[4] The broad band around $1305 \mathrm{~cm}^{-1}$ is related to the C-N and C- 
$\mathrm{N}+\bullet$ stretching modes, which all appeared in the spectrum of the nanoparticles containing a PANI.[2]

The bands at $1147 \mathrm{~cm}^{-1}$ corresponds to $-\mathrm{NH}^{+}$stretching modes of the doped state.[1]

The vibration peaks appearing in the PEDOT/PANI - JNPs FTIR spectrum, at 1354 and $1519 \mathrm{~cm}^{-}$

${ }^{1}$ are due to $\mathrm{C}=\mathrm{C}$ and $\mathrm{C}-\mathrm{C}$ stretching of the quinoidal structure of the thiophene ring.[5] The C-O-C bond stretching in the ethylene dioxy group can be observed in the peaks at 1147 and $1043 \mathrm{~cm}^{-1}$.[2] The peaks observed at 977 and $839 \mathrm{~cm}^{-1}$ it is C-S bond stretching in the thiophene ring.[2]

The FTIR spectra confirms that the surface of the support particles are covered by a PANI layer and PEDOT/PANI bilayer respectively.

\section{Confinement effects on the UV-vis-NIR absorption of the PEDOT/PANI layer}

The ultraviolet-visible-near-infrared (UV-vis-NIR) spectrum of the PEDOT/PANI layer in the homologous series of PEDOT/PANI-TESPN JNPs-3,-4,-5,-6 are presented in Figure S7. It is known from the literature that PANI UV-vis-NIR absorption spectrum can display two characteristic bands at about $366 \mathrm{~nm}$ and $733 \mathrm{~nm}$, which can be assigned to a $\pi-\pi^{*}$ transition of benzenoid ring and the benzenoid-quinoid excitonic transition, respectively.[6,7] The absorption at $733 \mathrm{~nm}$ is commonly attributed to the excitation of the quinone diimine structure in PANI. PEDOT can exist in three different states, depending on the polymer backbone oxidation state: PEDOT (neutral), $\mathrm{PEDOT}^{+}$(polaron) and $\mathrm{PEDOT}^{2+}$ (bipolaron). These states of PEDOT chains show absorption at different wavelengths: the neutral polymer chains show absorption around $600 \mathrm{~nm}$ is due to $\pi-\pi *$ transition; the chains in polaron state show absorption around $900 \mathrm{~nm}$ and the chains in bipolaron state show broad absorption in the infrared region (IR, from $1050 \mathrm{~nm}$ to higher wavelengths).[8-10] 
In the homologous series of PEDOT/PANI-TESPN JNPs-3,-4,-5,-6 with increasingly larger TESPN insulating lobe, the $\pi-\pi^{*}$ absorption bands have a substantial increase in intensity and show a red shift from 509 to $567 \mathrm{~nm}$ owing to a decrease in the band-gap energy (increase in conjugation length) as a result of increase in the thickness of PEDOT/PANI layer (Figure S7). At the same time the absorption of the polaron bands can be observed at around $830 \mathrm{~nm}$ decreases in intensity with the increase in the PEDOT/PANI layer thickness, which indicates a transition from a quinoidic structure of PEDOT into a more neutral state of the polymer backbone. It has been previously reported in mixed PEDOT/PANI layers that PANI has a doping effect on the PEDOT, with the net result of increasing the conductivity of the later.[11] For the confined PEDOT/PANI layers, the increase in the polaron band intensities with the decrease in layer thickness should indicate a higher level of mutual interaction between the conductive layers, sometimes referred to as "cooperative doping".[11] Meanwhile, all these peaks also show a red-shift 829 to 838 in the spectra of the PEDOT/PANI-TESPN JNPs, which could be ascribed to the interaction of PANI with PEDOT[2] with the increase in the thickness of layer of semiconductive polymers. This shift indicates the inter-chain interaction between PEDOT and PANI, which is believed to facilitate the formation of a conductive network that increases the electrical conductivity.[1] The correlation between conductivity and the thickness of conductive polymer was previously reported $[12,13]$ which increases by increasing the layer deposition or increase the thickness of PEDOT. 


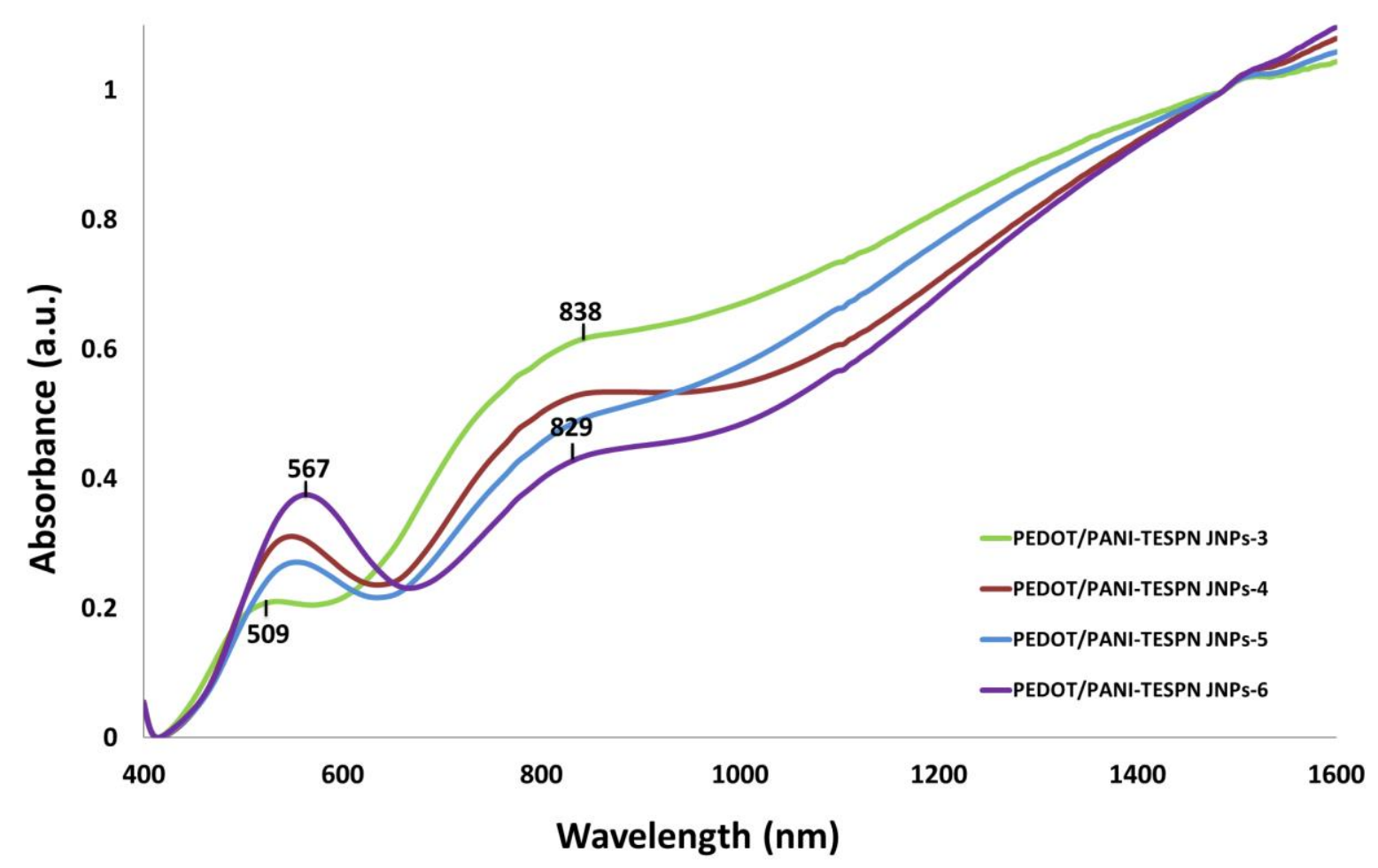

Figure S7. UV-vis-NIR absorption spectra of PEDOT/PANI-TESPN JNPs-3,-4,-5,-6.

\section{References}

[1] Y. Wang, S. Wu, Q. Yin, B. Jiang, S. Mo, Tuning thermoelectric performance of Poly(3,4-ethylenedioxythiophene): Poly (styrene sulfonate)/Polyaniline composite films by nanostructure evolution of polyaniline, Polym. Test. 94 (2021) 107017. https://doi.org/10.1016/j.polymertesting.2020.107017.

[2] L. Zhang, H. Peng, P.A. Kilmartin, C. Soeller, J. Travas-Sejdic, Poly(3,4ethylenedioxythiophene) and Polyaniline Bilayer Nanostructures with High Conductivity and Electrocatalytic Activity, Macromolecules. 41 (2008) 7671-7678. https://doi.org/10.1021/ma8013228.

[3] H.J. Jeon, Y. You, M.J. Yoon, J.H. Youk, Preparation of polyacrylonitrile nanoparticles via dispersion polymerization of acrylonitrile using a poly $(\mathrm{N}$-vinyl pyrrolidone $)$-cobalt complex in an aqueous system, Polymer. 52 (2011) 3905-3911. https://doi.org/10.1016/j.polymer.2011.07.022.

[4] M. Trchová, I. Šeděnková, J. Stejskal, In-situ polymerized polyaniline films 6. FTIR spectroscopic study of aniline polymerisation, Proc. Int. Conf. Sci. Technol. Synth. Met. 154 (2005) 1-4. https://doi.org/10.1016/j.synthmet.2005.07.001.

[5] D. Nandi, A.K. Ghosh, A. De, P. Sen, U.C. Ghosh, Fabrication, nanostructure evaluation, 3D electrical transport and electrochemical capacitance of PEDOT-Ti(IV)doped iron(III) oxide nanocomposite, J. Mater. Sci. 49 (2014) 776-785. https://doi.org/10.1007/s10853-013-7760-4.

[6] J. Kan, R. Lv, S. Zhang, Effect of ethanol on properties of electrochemically synthesized polyaniline, Synth. Met. 145 (2004) 37-42. https://doi.org/10.1016/j.synthmet.2004.04.017. 
[7] D. Li, Y. Jiang, Z. Wu, X. Chen, Y. Li, Fabrication of self-assembled polyaniline films by doping-induced deposition, Thin Solid Films. 360 (2000) 24-27. https://doi.org/10.1016/S0040-6090(99)00948-7.

[8] S.G. Im, K.K. Gleason, Systematic Control of the Electrical Conductivity of Poly(3,4ethylenedioxythiophene) via Oxidative Chemical Vapor Deposition, Macromolecules. 40 (2007) 6552-6556. https://doi.org/10.1021/ma0628477.

[9] O. Bubnova, Z.U. Khan, A. Malti, S. Braun, M. Fahlman, M. Berggren, X. Crispin, Optimization of the thermoelectric figure of merit in the conducting polymer poly $(3,4$ ethylenedioxythiophene), Nat. Mater. 10 (2011) 429-433. https://doi.org/10.1038/nmat3012.

[10] J. Wang, K. Cai, S. Shen, A facile chemical reduction approach for effectively tuning thermoelectric properties of PEDOT films, Org. Electron. 17 (2015) 151-158. https://doi.org/10.1016/j.orgel.2014.12.007.

[11] O.P. Dimitriev, Cooperative doping in polyaniline-poly(ethylene-3,4-dioxythiophene): poly(styrenesulfonic acid) composite system, J. Polym. Res. 18 (2011) 2435-2440. https://doi.org/10.1007/s10965-011-9657-8.

[12] Y. Kim, Y. Kim, J. Kim, Highly Conductive PEDOT:PSS Thin Films with TwoDimensional Lamellar Stacked Multi-Layers, Nanomaterials. 10 (2020) 2211. https://doi.org/10.3390/nano10112211.

[13] V.S. Sakkopoulos, E. Dalas, N. Paliatsas, K. Emmanouil, P. Malkaj, S.A. Choulis, A. Angelopoulos, T. Fildisis, Correlation between Thickness, Conductivity and Thermal Degradation Mechanisms of PEDOT:PSS Films, in: Alexandroupolis (Greece), 2010: pp. 178-181. https://doi.org/10.1063/1.3322407. 